\title{
Effectiveness of automated fuelsticks for predicting the moisture content of dead fuels in Eucalyptus forests
}

\author{
$\underline{\text { W. Bovill }}$, S. Hawthorne ${ }^{\mathrm{a}}$, J. Radic ${ }^{\mathrm{b}}$, C. Baillie ${ }^{\mathrm{a}}$, A. Ashton ${ }^{\mathrm{a}}$, P. Noske ${ }^{\mathrm{a}}$, P. Lane ${ }^{\mathrm{a}}$ and G. Sheridan ${ }^{\mathrm{a}, \mathrm{c}}$ \\ ${ }^{a}$ Forest Hydrology Research Group, School of Ecosystem and Forest Sciences, Faculty of Science, The \\ University of Melbourne, Victoria. \\ ${ }^{b}$ Victorian Department of Environment, Land, Water and Planning. \\ ${ }^{c}$ Bushfire and Natural Hazards Cooperative Research Centre, 340 Albert Street, East Melbourne, Victoria \\ 3002, Australia. \\ Email: sheridan@unimelb.edu.au
}

\begin{abstract}
The fuel moisture content (FMC) of forest litter strongly affects fire ignition and behaviour, and is a key factor in planning when and where to burn. Planned burns are safest and most efficient when FMC lies within a narrow range $(9 \%-17 \%)$. Drier fuels $(<9 \% \mathrm{FMC})$ can burn too intensely and fires may be difficult to control, while wetter fuels (>17\% FMC) may fail to ignite and/or burn poorly. Planned burning is associated with real risks of escape, and large annual operational costs (e.g. in the order of several \$100M's per year in several Australian states), but in the absence of reliable estimates of FMC, fire behaviour predictions are compromised, and many burning opportunities may be missed, leading to the inefficient allocation of resources and compromising strategic burn planning.

The aim of this research is to quantitatively evaluate the performance of Campbell Scientific 10-hour fuel moisture sticks for the prediction of the FMC of dead surface and elevated fuels in a wide variety of Eucalyptus forests. Fuelsticks can be installed and monitored remotely, providing continuous real-time information to fire managers on fuel conditions. The performance of the sensors was evaluated in the context of the needs of planned burn managers, specifically, i) daily FMC trend information to assist in the efficient scheduling and resource allocation in the weeks and days prior to the burn, and ii) hourly FMC prediction to build an understanding of how FMC is responding to current conditions. The primary research objectives were to, a) quantify the relationship between Campbell 10-hour fuel moisture stick readings and the in situ gravimetric FMC of surface and elevated fuels collected from $40 \mathrm{~m}$ x $40 \mathrm{~m}$ plots, b) use the collected data to develop site-specific corrections to improve fuelstick FMC predictions, and c) evaluate the capacity of the uncorrected and corrected fuel moisture stick FMC readings to improve operational planned burning decision making. The fuelsticks were installed in eight contrasting forested locations across south east Australia and evaluated from December 2014 to June 2015.
\end{abstract}

The results showed that the uncorrected 10-hour fuelstick readings resulted in prediction errors of sufficient magnitude (RMSE of 8-17\% FMC) that limits the utility of this method (as applied in this study) for planned burning decision making at the scale of days to weeks, resulting in correct planning decisions only about $50 \%$ of the time. However with site-specific corrections and the use of addition microclimate data the fuelsticks enabled correct planning decisions $75 \%$ of the time at the scale of days to weeks. Additional fuelstick replication and the use of fuelsticks with shorter response times could further improve the predictive ability of this method. At sub-daily scales the 10-hour fuelsticks were poorly suited predicting current FMC conditions, as the FMC of fuels changed more rapidly than FMC of fuelsticks. The results also indicated that the use of EMC type models to predict the FMC of the surface and profile litter is likely to be unsuccessful due to slow response time of the fuels relative to the rate of change of the atmospheric forcing during the day, and due to the lack of representation of recent precipitation on FMC.

Overall, these initial results indicate that locally-calibrated remotely monitored fuelsticks can be used to identify FMC trends which substantially improve planned burning decision making in the weeks and days prior to burning. However the 10-hour fuelsticks tested were unsuited to prediction at sub-daily scales, and more generally, further refinement of the fuelstick and microclimate FMC monitoring methodology is recommended in order to fully exploit the potential of this technology to improve operational decision making.

Keywords: $\quad$ Fuel moisture content, Campbell's fuel moisture sticks, fire, planned burning 


\section{INTRODUCTION}

Fuel moisture strongly affects fire ignition and behaviour, and is a key factor in planning when and where to burn. Planned burns are safest and most efficient when FMC lies within a narrow range $(9 \%-17 \%)$ (Slijepcevic et al. 2015). Drier fuels ( $<9 \%$ FMC) can burn too intensely and fires may be difficult to control, while wetter fuels ( $>17 \%$ FMC) may fail to ignite and/or burn poorly, failing to meet fuel reduction targets. In Victoria fuel moisture estimates for burn scheduling are historically based on weather observations and the deployment of field staff to take manual FMC measurements. This process is prone to substantial prediction error, resulting in both missed burning opportunities, and inefficient scheduling and allocation of resources. Furthermore, these manual point estimates of FMC are difficult to summarise when reporting to government on how the burning program was aided or hindered by fuel moisture conditions throughout a season.

To overcome these limitations, the Victorian Department of Environment, Land, Water and Planning (DELWP) proposes an 'Automated Fuel Moisture Monitoring Network' (AFMMN) of 50 sites with fuel moisture sensors (\#22601 10 hour Fuel Moisture Sticks, Campbell Scientific, Inc., Logan, USA) to provide a continuous, state-wide overview of fuel moisture conditions that is accessible to DELWP staff online via the FireWeb (Radic 2014). Pine fuelsticks are used extensively in fuel moisture monitoring programs in the United States and Canada (e.g. see NWCG 2012), and have been used in Australia (e.g. Marsden-Smedley and Catchpole 2001) but, to our knowledge, not operationally. It is proposed that access to reliable real-time FMC data will cut labour costs associated with regular site visits, and enable staff to better identify and forecast suitable burn-windows. The result should be fewer missed burning opportunities and reduced undue mobilisation of resources when conditions are not suitable. The AFMMN is to be established in four phases, 1) equipment test, 2) operational trial, 3) detailed evaluation, and 4) full network rollout. The work described in this paper is associated with Phase 3 of this program.

\section{AIM}

The aim of this research is to quantitatively evaluate the performance of Campbell Scientific 10-hour fuel moisture sticks for the prediction of the FMC of dead surface fuels and elevated fuels in Victorian forests. The performance of the sensors will be evaluated in the context of the needs of planned burn managers, specifically,

1. Daily samples collected during wetting/drying cycles to provide spatially representative estimates of FMC values over a range of conditions (dry to wet) to compare with averaged estimates of FMC from the fuelstick during the same periods.

2. Hourly samples from 9:00am - 5:00pm to compare the change in the fuelstick and gravimetric FMC during a single day.

The primary research objectives are:

1. To quantify the relationship between Campbell 10-hour fuel moisture stick hourly readings and the in situ gravimetric FMC of dead fine surface fuels and elevated fuels.

2. To use the collected data to develop site-specific corrections.

3. To evaluate the capacity of the uncorrected and corrected fuel moisture stick FMC readings to improve operational planned burning decision making.

\section{METHODS}

\section{1. $\quad$ FMC and microclimate monitoring sites}

Eight fuelstick and microclimate monitoring sites were established in contrasting forest types across a large climate gradient in Victoria, Australia (shown in red in Figure 1). The sites were monitored for a seven month period from December 2014 to June 2015. In this study the four sticks were deployed in a vertical array (but not directly beneath one another) at four heights: 'Soil contact' sticks were placed beneath litter, in contact with the soil; 'Exposed' sticks were placed on top of the litter, exposed to the air; 'Near Surface' sticks were suspended $10 \mathrm{~cm}$ above ground; 'Elevated' sticks were suspended $50 \mathrm{~cm}$ above ground. The fuelsticks are constructed from ponderosa pine dowels (diameter: $13 \mathrm{~mm}$, length: $508 \mathrm{~mm}$, weight: $45 \mathrm{~g}$ ) with electrodes embedded along opposite sides. A fuel moisture sensor (CS506, Campbell Scientific, Inc., Logan, USA) is attached to each stick and converts conductivity between the electrodes into a FMC reading, which is logged every 15 minutes. The CS506 moisture sensor has an operating range of 0 to $70 \%$ moisture content, and sensor error of $\pm 0.74 \%(0-10 \% \mathrm{FMC})$ to $\pm 2.27 \%$ (30-50\% FMC). 


\subsection{Gravimetric field FMC sampling}

Sampling plots $(40 \mathrm{~m} \times 40 \mathrm{~m})$ were established at each site to sample gravimetric FMC. A $1600 \mathrm{~m}^{2}$ plot provided sufficient space for the high intensity, destructive sampling program (up to 240 random quadrats per site). The fuel moisture sticks and weather station were located centrally within each site. A $4 \mathrm{~m} \times 4 \mathrm{~m}$ area surrounding the equipment was excluded from gravimetric sampling due to high traffic of personnel downloading loggers and servicing equipment. Sampling for gravimetric FMC was conducted at two timescales:

1. Daily samples collected during wetting/drying cycles to provide point estimates of a range of FMC values (dry to

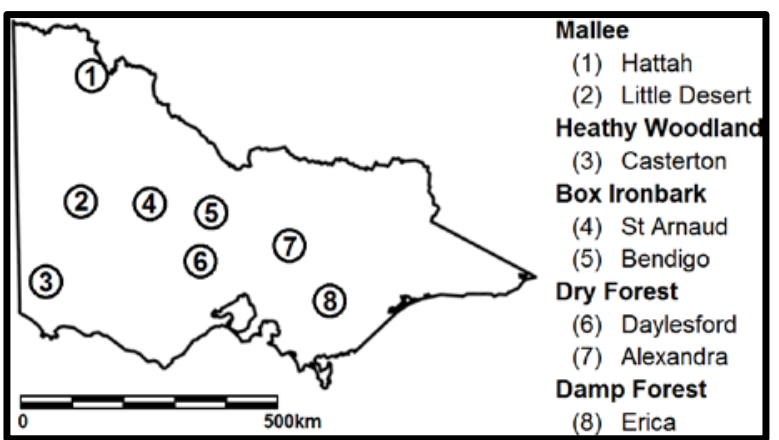

Figure 1. The location of the eight trial sites evaluated in this study (red stars), spanning a wide geographical range and variety of vegetation types. wet) to compare with point estimates of FMC from the fuelstick.

2. Hourly samples from 9:00am - 5:00pm to compare the change in the fuelstick and gravimetric FMC during the day.

Four different fuel types were sampled at each site;

1. Surface litter - the top $1 \mathrm{~cm}$ of litter profile.

2. Sub-surface litter - the remainder of litter profile down to the mineral soil surface.

3. Profile litter - both the surface and sub-surface litter combined.

4. Elevated fuels - dead fuels including twigs, leaves, bark, bracken, grasses and spinifex collected from about $50-100 \mathrm{~cm}$ above ground.

Surface and sub-surface fuels correspond with the "exposed" and "soil contact" fuel moisture sticks, respectively, and elevated fuels may correspond best with "elevated" fuelstick. Samples of surface and subsurface fuels were collected from 15 randomly located quadrats per site. Samples were collected within a short time $(\leq 1.5 \mathrm{~h})$ to avoid error introduced by fluctuations in FMC. Fuelstick readings during this period were averaged for comparison with the gravimetric measurements. surface litter was removed from the quadrat into ziplock bags. Samples (one large handful) of elevated fuels were collected if such fuels occurred within a $2 \mathrm{~m}$ radius of a quadrat. Samples from all quadrats were pooled to

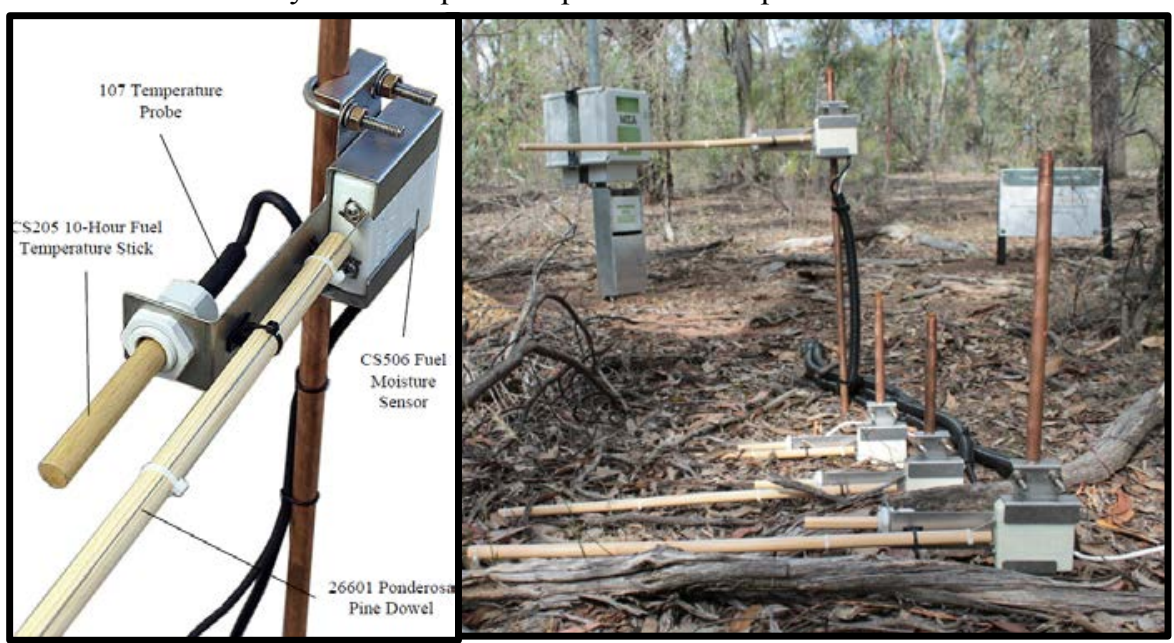

Figure 2. (Left) Standard setup of 10-hour Fuel Moisture Stick and sensor as offered by Campbell Scientific, paired with 10-hour Fuel Temperature Stick and sensor (source: www.campbellsci.com.au). (Right) An example of one of the fuelstick and microclimate monitoring stations, showing four Campbell Scientific 10-hour fuelsticks and adjacent temperature sensors installed at different heights. give one composite sample for each fuel type per site. The wet weight of all samples was measured in the field to two decimal places, and the dry weights were determined in the laboratory after the oven drying samples for 24 hours at $105^{\circ} \mathrm{C}$. On one day per site, samples of surface and sub-surface fuels were collected hourly (09:00 to 17:00), to compare changes in sensor and gravimetric FMC during the day. Each hourly sample was collected within the first 15 minutes of the hour to ensure a sufficient time-step between samples. To complete the sampling within this restricted timeframe only five quadrats of surface and subsurface fuel were sampled. 


\subsection{Analysis and interpretation}

The error associated with the prediction of the FMC of litter using fuelsticks was quantified using the root mean square error (RMSE), an error metric that is in the same units as the predicted and observed values. i.e. \% FMC). For each fuel type, the RMSE was calculated both for all the sites separately, and for all the data combined. Individual linear regressions (forced through the origin) were fitted to the data for each site, and the slope parameter $m$ was then used to as a site-specific correction factor. The RMSE of the corrected predictions was then calculated. To evaluate the impact of the model prediction error on planned burn decision making, we adopted the approach of Slijepcevic et al. (2015). Correct FMC predictions result in correct operational decisions, while incorrect predictions result in wasted time, patchy burns, or missed opportunities to burn (Figure 3 ). The FMC categories are $<9 \%$ too dry, $9-17 \%$ good, $>17-30 \%$ damp, and $>30 \%$ too wet (Figure 3 ).

\section{RESULTS}

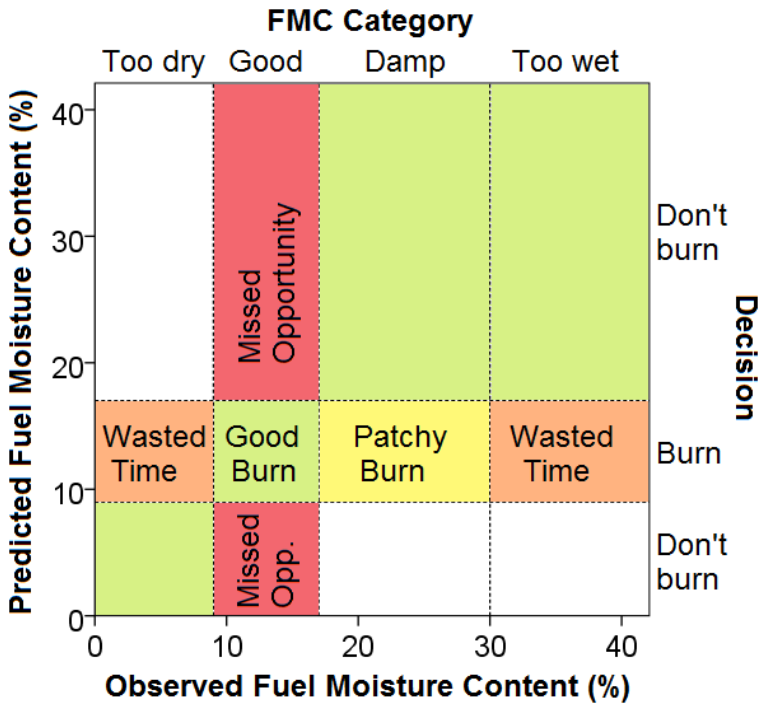

Figure 3. Operational consequences of decisions based on predicted (fuelstick) FMC. Correct decisions (green cells) fall along the 1:1 line. Figure adapted from Slijepcevic et al. (2015).

\subsection{Daily FMC sampling}

Preliminary analysis of the fuelstick data (not shown here) indicated that, of the four fuelstick installation positions (soil contact, exposed, near surface, and elevated), the "exposed" fuelstick in contact with the surface litter provided the most consistent results for the daily data. All of the daily analysis is based on this fuelstick installation position. The results from the daily sampling are shown in Figure 4 (excluding the Erica site due to equipment failure). The results indicate that the fuelstick generally results in an overestimate of the true FMC, with RMSEs for all the sites combined of $\pm 10,8$, and 17\% FMC for surface, profile and elevated fuels, respectively (Table 1). However there is considerable variability in the performance between different forest sites. For example, in the case of surface FMC, the fuelsticks are very effective at the Bendigo site $(m=1.03$, RMSE $=2.5 \%)$, yet perform poorly at the St Arnaud site $(m=0.65$, RMSE $=13 \%)$. Elevated fuels (Figure 4c) are particularly poorly predicted by the fuelstick across all the sites (RMSE of $17 \%$ for all sites combined).

The application of site-specific correction factor $m$, the slope of a linear model with no intercept (see Figure 4 a-c), improves the predictive capacity of the fuelstick to varying degrees (Figure $4 \mathrm{~d}$-f and Table 1). Across all the sites, application of the correction factors reduces the RMSE from 10 to $6 \%$ FMC for the surface fuel, from 8 to $7 \%$ FMC for the profile fuel, and from 17 to $7 \%$ FMC for the elevated fuel. 
Bovill et al., Effectiveness of automated fuelsticks for predicting fuel moisture
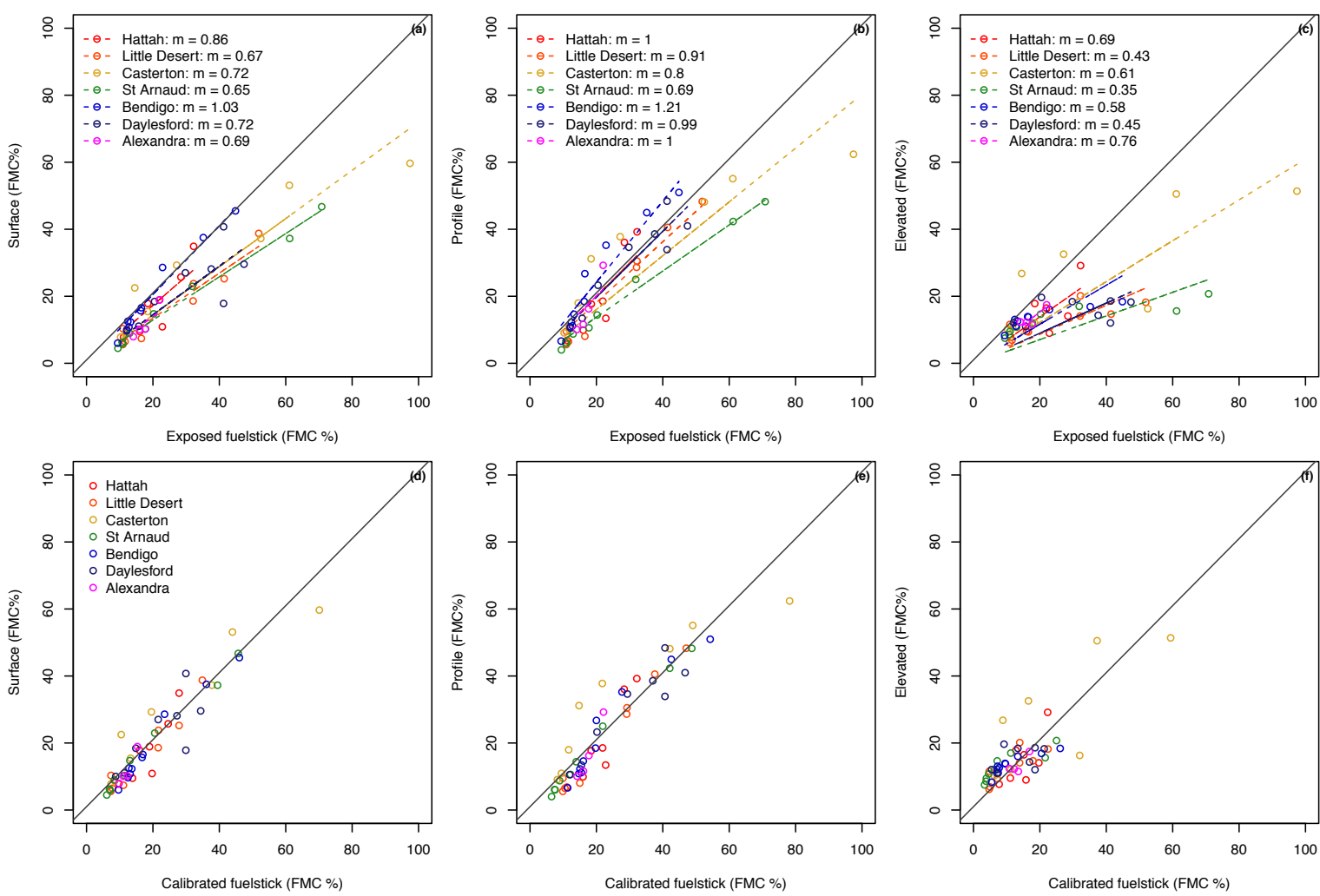

Figure 4. Relationship between the exposed fuelstick fuel moisture content (FMC) and the observed gravimetric FMC. Figures a \& d show surface $(<1 \mathrm{~cm}$ deep) fuels only, Figures b \& e show the FMC of the

entire litter profile, and Figures c \& f show elevated fuels only. Figures a, b and c show the raw, un-

calibrated values from the fuel sticks. Figures d, e \& f show the fuelstick values following the application of a site-specific correction factor.

Table 1. Root mean square error (RMSE) values ( $\pm \mathrm{x} \%$ FMC) of the prediction models for surface, profile and elevated FMC. "Raw" refers to the RMSE calculated with no site-specific correction, while "corrected" refers to the RMSE calculated from a linear model with no intercept for each site. The final row shows the percentage of correct FMC category classifications based on the fuelstick predictions (see Figure 3), with and without site-specific corrections.

\begin{tabular}{|c|c|c|c|c|c|c|}
\hline \multirow{3}{*}{ Site names } & \multicolumn{6}{|c|}{ Fuel type } \\
\hline & \multicolumn{2}{|c|}{ Surface } & \multicolumn{2}{|c|}{ Profile } & \multicolumn{2}{|c|}{ Elevated } \\
\hline & Raw & Corrected & Raw & Corrected & Raw & Corrected \\
\hline Hattah & 6 & 5 & 6 & 6 & 8 & 5 \\
\hline Little Desert & 10 & 3 & 4 & 4 & 17 & 4 \\
\hline Casterton & 15 & 7 & 14 & 11 & 22 & 12 \\
\hline St. Arnaud & 13 & 2 & 11 & 2 & 23 & 6 \\
\hline Bendigo & 3 & 2 & 6 & 4 & 11 & 5 \\
\hline Daylesford & 11 & 6 & 5 & 5 & 19 & 6 \\
\hline Alexandra & 6 & 2 & 5 & 5 & 4 & 1 \\
\hline All sites combined & 10 & 6 & 8 & 7 & 17 & 7 \\
\hline $\begin{array}{l}\% \text { of correct FMC } \\
\text { classifications (i.e. } \\
\text { too dry, good, damp, } \\
\text { too wet) }\end{array}$ & 54 & 74 & 57 & 72 & 43 & 48 \\
\hline
\end{tabular}




\subsection{Microclimate predictors of FMC}

In some cases microclimate data collected at the monitoring site could be used to better predict FMC instead of the fuelstick. Figure 5c shows that vapour pressure deficit (VPD), a function of relative humidity and temperature, is a strong predictor of elevated FMC, even in the absence of site specific corrections. However VPD is a poor predictor of surface or profile FMC (Figures $5 \mathrm{a}$ and $\mathrm{b}$ ).
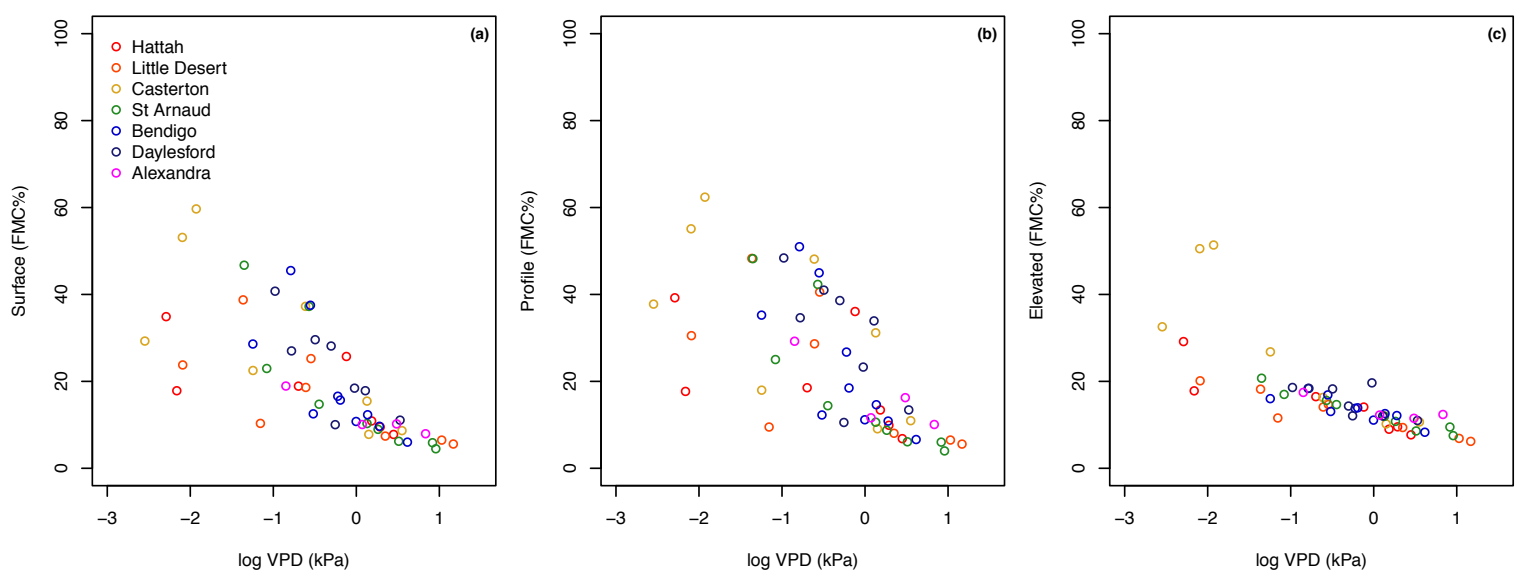

Figure 5. Relationship between the natural log of the vapour pressure deficit (VPD) at screen height, and gravimetric fuel moisture content (FMC) of (a) surface ( $<1 \mathrm{~cm}$ deep) fuels, (b) entire litter profile and (c) elevated dead fuels at seven sites. Each point represents sampling on a different day.

\subsection{Hourly FMC sampling}

The results from the hourly FMC sampling are shown in Figure 6. Note that the fuelstick values in Figure 6 reflect the raw data; no site specific corrections have been applied. The differences between the FMC predicted from the fuelsticks, and the measured gravimetric FMC are large; in the order of $5-15 \%$ FMC. These differences are variable across the monitored sites making generalizations difficult, however some common patterns were observed. Firstly, the litter (surface and sub-surface) dries out substantially faster than the fuelsticks in most cases, resulting in large (5-10\%) overestimates of FMC by noon. This can be clearly seen for the St Arnaud and Daylesford timeseries.

\section{DISCUSSION}

The raw (i.e. with no site-specific corrections) fuelstick data provide only a moderate level of predictive capacity, with high RMSE relative to the narrow FMC window for "good" burns (9-17\%) This predictive error results in the incorrect categorisation of FMC (i.e. too dry, good, damp, too wet) using the fuelstick about $50 \%$ of the time, meaning that the use of raw data will frequently result in costly missed opportunities or wasted resources.. Site-specific single-parameter corrections reduced RMSE considerably, increasing the proportion of correct categorisations and resulting in correct burn planning decisions about $75 \%$ of the time for surface and profile fuels.

The remaining predictive uncertainty could be attributed to i) lack of replication of the fuelsticks to account for spatial variations in FMC within sites, ii) inherent sensor and sampling errors iii) different drying-phase response times of the 10-hour fuelstick and the fine fuels, resulting in misaligned drying curves of predicted and observed FMC over short (e.g. sub-daily) timescales. Unfortunately, fuel moisture models based on humidity, temperature, and VPD measurements (a cost effective alternative to fuelsticks that require installation, calibration, maintenance, and replacement) gave poor prediction of surface and profile FMC. Nevertheless, the VPD models can be used to monitor the FMC of elevated fuels, eliminating the requirement for 'near surface' and 'elevated' fuelsticks in the AFMMN. Account-keeping type water balance models using additional micro-climate data such as rainfall and dew inputs may be better suited to the prediction of the FMC of the surface and profile fuels. 


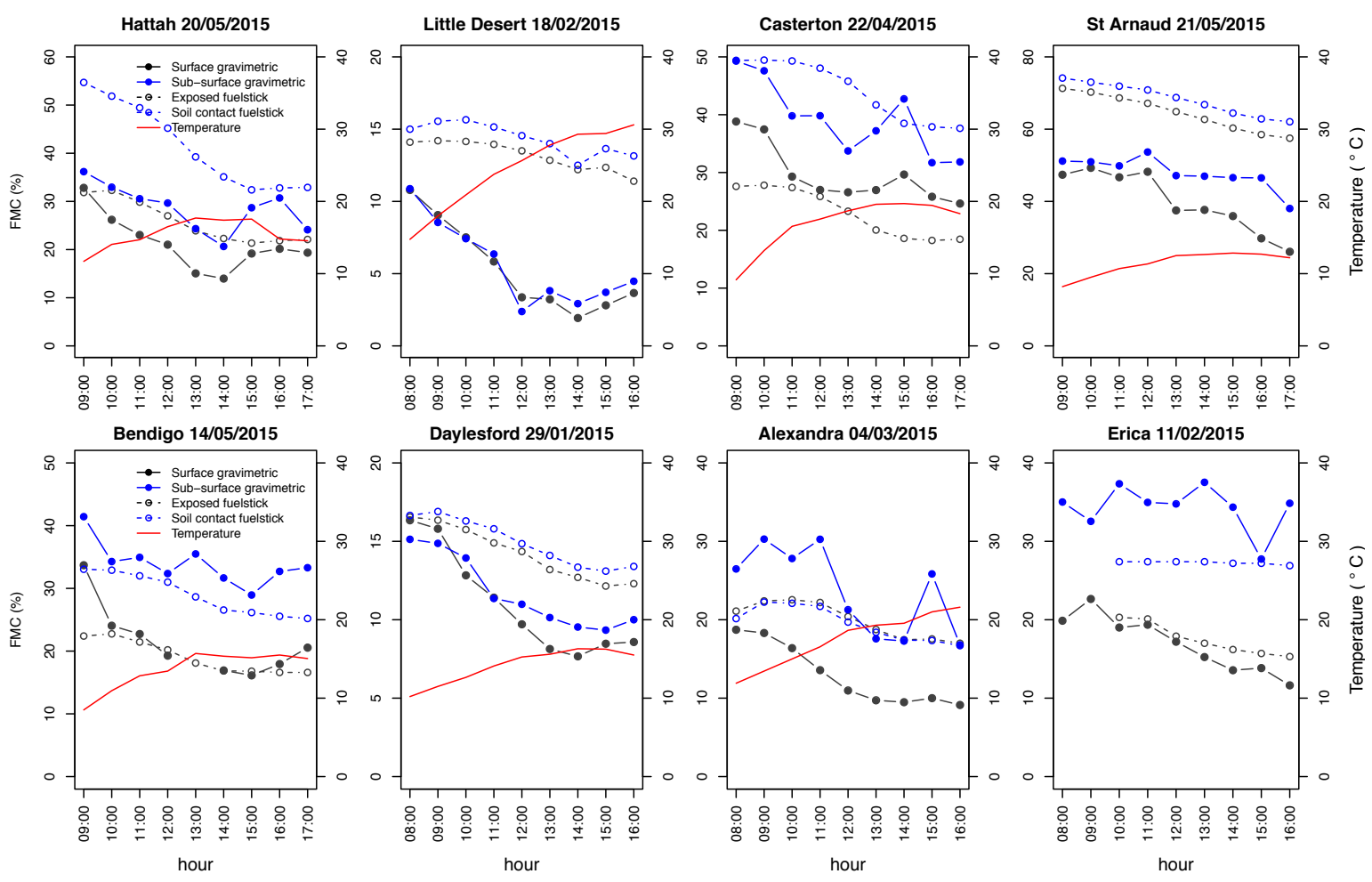

Figure 6. A comparison between the hourly predicted fuel moisture content (FMC) using 10-hour fuelsticks installed in contact with the soil (blue dashed line) and in contact with the surface of the litter (black dashed line), and the gravimetrically measured FMC of the surface litter fuels $<1 \mathrm{~cm}$ deep (black solid line) and the sub-surface litter $>1 \mathrm{~cm}$ deep (blue solid line), for 8 different field locations. Hourly temperature change is also shown.

\section{CONCLUSION}

Site-corrected fuelstick data enabled correct planning decisions $75 \%$ of the time. The 10 -hour fuelsticks were poorly suited to the identification of sub-daily "burn-windows" due to the fuels being more responsive than the fuelsticks, resulting in large prediction errors as the day progressed. The data indicate that there is considerable potential to further refine the fuelstick and microclimate FMC monitoring methodology so as to fully exploit the potential of this technology to improve operational decision making.

\section{ACKNOWLEDGMENTS}

The authors would like to acknowledge DELWP for providing financial assistance for this research. We would also like to thank the two anonymous reviewers for their valuable comments during the review process.

\section{REFERENCES}

Marsden-Smedley, J. B. and W. R. Catchpole (2001). Fire modelling in Tasmanian buttongrass moorlands. III - Dead fuel moisture. International Journal of Wildland Fire 10(2): 241-253.

Matthews, S. (2014). Dead fuel moisture research: 1991-2012. International Journal of Wildland Fire 23(1): 78-92.

NWCG (2012). Interagency Wildland Fire Weather Station Standards \& guidelines, National Wildfire Coordinating Group.

Radic, J. (2014). Business Case: Automated Fuel Moisture Monitoring Network (V.3). DEPI.

Slijepcevic A., Anderson W.R., Matthews S. and Anderson D.H. (2015). Evaluating models to predict daily fine fuel moisture content in eucalypt forest Forest Ecology and Management 261: 261-269. 